\title{
Mittheilungen über Pepsin.
}

\author{
Von
}

C. A. Pekelharing.

(Der Redaction zugegangen am 6. Februar 1902.)

Vor einigen Jahren habe ich mitgetheilt, ${ }^{1}$ ) dass sich aus künstlichem Magensaft ein eigenthümlicher Eiweissstoff bereiten lässt, welcher in stark verdünnter Säure $(0,02 \% \mathrm{HCl})$ schwer löslich ist und in auffallendem Maasse die eiweissverdauende Wirkung des Pepsins besitzt. Ich habe damals die Vermuthung ausgesprochen, diese Substanz sei vielleicht nicht mit Pepsin gemischtes Eiweiss, sondern das Enzym selbst. Ich war aber nicht im Stande, dieser Vermuthung einen festen Boden zu geben, weil es mir nicht gelungen war, jenen Eiweissstoff von Beimischungen zu befreien. Besonders war der Gehalt an Phosphor schwankend, gewöhnlich ungefähr $1 \%$, bisweilen aber auch viel geringer. "Ich glaube,» so schrieb ich, «einigen Grund für die Annahme zu haben, dass das Pepsin bald mehr, bald weniger mit einer wasserlöslichen P-haltigen Substanz verunreinigt sei. Ist das thatsächlich der Fall, so wird diese Verunreinigung durch nochmalige Wiederholung des Lösens in Salzsäure und Fällen mittelst Dialyse zu beseitigen sein. Diese Operation geht aber mit grossem Verlust an Material einher, da die Fällung mittelst Dialyse immer unvollständig ist.»

Die Gruppe der unter dem Namen «Enzyme» zusammengefassten Stoffe ist noch immer von einem geheimnissvollen Schleier verhüllt. Ich glaube durch frühere Untersuchungen dargethan zu haben, dass das Fibrinferment zu den Eiweiss-

1) Diese Zeitschr., Bd. XXII, S. 233. 
stoffen, und zwar zu den Nucleoproteiden zu rechnen sei. Dieser Stoff wird aber vielleicht nicht von Allen als ein echtes Enzym betrachtet. Jedenfalls ist das Fibrinferment nicht immer dieselbe Substanz, da nicht nur aus dem Blut, sondern auch aus verschiedenen Organen Nucleoproteide, welche alle die Wirkung des Fibrinferments zeigen, bereitet werden können. Deshalb kam es mir der Mühe werth vor, die Vermuthung, auch das Pepsin, welches gewiss allgemein als ein typisches Enzym betrachtet wird, gehöre zu den Eiweissstoffen, näher zu prüfen. Mit Rücksicht auf die grosse Unsicherheit, welche über die Natur der Enzyme herrscht, und auf den wiederholt von hervorragenden Forschern ausgesprochenen Zweifel an der Zusammengehörigkeit dieser mysteriösen Stoffe mit den Eiweissstoffen, war aber die grösste Umsicht bei der Arbeit, welche voraussichtlich viel Zeit und Mühe in Anspruch nehmen würde, geboten.

An erster Stelle hatte ich zu untersuchen, ob es möglich wäre, den fraglichen Stoff in solcher Reinheit aus der Magenschleimhaut $\mathrm{zu}$ bereiten, dass man berechtigt sein könnte, denselben als ein chemisches Individuum zu betrachten.

Trotz lange fortgesetzter Versuche ist es mir nicht gelungen, einen Stoff von unveränderlicher Zusammensetzung aus der Magenschleimhaut zu erhalten. Einige Hunderte von Schweinemagen habe ich verarbeitet und daraus den fraglichen Stoff nach wiederholter Reinigung in zur Analyse genügender Menge bereitet. Der $\mathrm{N}$ - und $\mathrm{H}-$ Gehalt war in den verschiedenen Präparaten ziemlich gleich, der C- und besonders der P-Gehalt zeigten aber Schwankungen, welche auf Verunreinigung zu schliessen nöthigten. Je weiter die Reinigung fortgesetzt wurde, desto kleiner fand ich im Allgemeinen den P-Gehalt; die niedrigste dafür gefundene Zahl war $0,425 \%$.

Die Bereitungsweise war in der Hauptsache die früher beschriebene. Ein Theil des Pepsins wurde aus der klar filtrirten Digestionsflüssigkeit mittelst Dialyse gegen strömendes Wasser ausgefällt und auf der Centrifuge abgetrennt. Dann wurde die Lösung mit basischem Bleiacetat und Ammoniak behandelt, der Niederschlag mit Oxalsäure zersetzt und die vom Bleioxalat 
filtrirte Flüssigkeit dialysirt. Das hierbei ausgetällte Pepsin wurde mit der zuerst ausgeschiedenen Portion vereinigt, in möglichst wenig $0,2 \%$ iger Salzsäure bei $37^{\circ} \mathrm{C}$. gelöst und bei derselben Temperatur filtrirt. Die völlig klare, gelblich gefärbte Lösung wurde in die 8-10fache Menge destillirten Wassers ausgegossen und vorsichtig mit äusserst verdünnter Natron- oder Kalilauge versetzt, bis empfindliches Congopapier nicht mehr gebläut wurde. Zur möglichst vollständigen Ausscheidung des Pepsins blieb die Flüssigkeit eine Nacht über im Eisschrank. Dann wurde sie centrifugirt. Der Niederschlag wurde bei $37^{\circ} \mathrm{C}$. in möglichst wenig 0,20 \%iger $\mathrm{HCl}$ gelöst. Die nahezu farblose Lösung wurde bei $37^{\circ} \mathrm{C}$. filtrirt und dann in einem kleinen Dialysatorschlauch in destillirtes Wasser gestellt. Im Dialysator setzte dann das Pepsin sich in kleinen, gruppenweise zusammenhaftenden, durchsichtigen, ziemlich stark lichtbrechenden Kügelchen ab. Nach etwa 20 stündigem Dialysiren wurde dieser Niederschlag abfiltrirt, einmal mit destillirtem Wasser übergossen und nach vorsichtigem Auspressen des Filters zwischen Filtrirpapier vom Filter abgehoben und über Schwefelsäure oder Chlorcalcium bei Zimmertemperatur getrocknet. Wenn der Niederschlag gehörig ausgepresst ist, bildet derselbe eine zusammenhängende Schicht, welche leicht, ohne dass Papierfasern mitgehen, vom Filter abgehoben werden kann. Das so bereitete Pepsin ist schwach gelblich gefärbt und wird beim Trocknen dunkler. Fein zerrieben, stellt es ein aschefarbiges, nicht oder kaum hygroskopisches Pulver dar. Wie oft ich die Lösung in Salzsäure und die Fällung mittelst Dialyse auch wiederholt habe, nie erhielt ich ein farbloses Präparat.

Die vom Bleioxalat abfiltrirte, dialysirte und dann vom ausgeschiedenen Pepsin befreite Lösung hat immer noch eine ansehnliche verdauende Kraft; sie enthält noch eine beträchtliche Menge Pepsin. Diese Lösung wurde nun mit Ammonsulfat gesättigt. Sobald die Sättigung vollständig ist, setzt sich der dadurch verursachte Niederschlag in klebrigen, leicht $\mathrm{zu}$ filtrirenden Flocken ab. Das klare, farblose Filtrat gibt starke Biuretreaction, ist aber frei von Pepsin; mittelst Dialyse vom Ammonsulfat befreit, zeigt es, nach Salzsäurezusatz bis zu 
einem Gehalt von $0,2 \%$, keine Spur von verdauender Wirkung. Der Niederschlag lässt sich als eine zähe, zusammenhängende Masse leicht vom (gehärteten) Filter abnehmen und wird im feuchten Zustand, ohne Zusatz von Wasser, in einen Dialysatorschlauch gebracht, welcher in strömendem Wasser aufgehängt wird. Das Wasser dringt in den Schlauch hinein und löst den Niederschlag innerhalb 24 Stunden zum grössten Theil. In dieser Weise wird das Pepsin erstens von einem Theil der Verdauungsprodukte der Magenschleimhaut und vom zugesetzten Ammonsulfat befreit, dann aber, und das ist wichtiger, in concentrirtere Lösung gebracht. Indem die vom Bleioxalat abfiltrirte und dann mittelst Dialyse von einem Theil des Pepsins befreite Flüssigkeit ein Volum hatte von etwa $300 \mathrm{ccm}$., ist jetzt nach der Dialyse der mit Ammonsulfat erhaltenen Fällung die ganze noch übrig gebliebene Pepsinmenge in etwa $80 \mathrm{ccm}$. Flüssigkeit gelöst. Diese Flüssigkeit wird nun mit Salzsäure versetzt bis zu einem Gehalt von $0,02 \% \mathrm{HCl}$, zur Beförderung der Ausscheidung und zur Entfernung des noch vorhandenen Salzes einen Tag lang gegen Salzsäure derselben Concentration bei einer Temperatur nicht weit über $0^{\circ} \mathrm{C}$. dialysirt und dann abgesaugt. So wird wieder eine beträchtliche Menge Pepsin erhalten, welches ganz wie oben beschrieben behandelt wird: Auflösen in 0,2\%iger $\mathrm{HCl}$ bei $37^{\circ} \mathrm{C}$., Filtriren, Ausgiessen in das 8-10 fache Volumen Wasser, Zusatz von Alkali, bis Congopapier nicht mehr gebläut wird, Centrifugiren, Lösen in 0,20 ! 0 iger $\mathrm{HCl} u$. s. w. Schliesslich wird die gereinigte Substanz im Exsiccator bei Zimmertemperatur getrocknet und zerrieben.

So wurden aus den Schleimhäuten von je 10 Schweinemagen zwei ziemlich gleich grosse Portionen Pepsin erhalten, jede zu etwa 150 bis $200 \mathrm{mg}$.

Zwischen dem mittelst Ammonsulfat gefällten und dem zuvor mittelst Dialyse der Verdauungsflüssigkeit und mittelst der Bleifällung erhaltenen Pepsin habe ich keinen Unterschied finden können; auch nicht in Bezug auf die verdauende Kraft. Immer war 0,001 mg der bei Zimmertemperatur im Exsiccator getrockneten Substanz im Stande, bei $37^{\circ} \mathrm{C}$. eine Fibrinflocke 
in $6 \mathrm{ccm} .0,2 \%$ ige $\mathrm{HCl}$ innerhalb etwa 20 Stunden zu lösen. Dieser Versuch wurde nie ohne Kontrolle angestellt, in dieser Weise, dass in demselben Wasserbad eine Fibrinflocke mit 6. ccm. 0,2\% iger $\mathrm{HCl}$ ohne Pepsin digerirt wurde. Der Unterschied zwischen den beiden Proben war immer schon nach wenigen Stunden durchaus beweisend.

Die ,von dem mittelst Dialyse gegen destillirtes Wasser gefällten Pepsin abfiltrirte Flüssigkeit enthält noch immer eine beträchtliche Menge des Enzyms in Auflösung; sie verdaut Eiweiss kräftig und gibt einen Niederschlag beim Erhitzen. Während nun aus den mit anderen Stoffen, hauptsächlich Verdauungsprodukten, stark verunreinigten Pepsinlösungen das Pepsin erst so gefällt wird von Ammonsulfat, dass es abfiltrirt werden kann, wenn die Flüssigkeit mit dem Salz gesättigt ist, bildet sich in jener nahezu reinen Pepsinlösung schon bei halber Sättigung mit Ammonsulfat ein gut filtrirbarer Niederschlag. Die Filtrate des gereinigten Pepsins wurden je unter Zusatz von $35 \mathrm{~g}$ Ammonsulfat auf $100 \mathrm{ccm}$. Flüssigkeit gesammelt und, sobald eine genügende Menge zusammengebracht. war, abfiltrirt. Der Niederschlag wurde abgepresst, mittelst Dialyse vom Salz befreit, bei $37^{\circ}$ in möglichst wenig $0,2 \%$ iger HCl gelöst, bei derselben Temperatur filtrirt, mittelst Dialyse wieder gefällt, abfiltrirt und getrocknet. So wurde aufs Neue Pepsin erhalten, dessen Eigenschaften vollkommen mit denjenigen des in der oben beschriebenen Weise bereiteten übereinstimmten. Auch von diesem Pepsin brachte $0,001 \mathrm{mg}$ in $6 \mathrm{ccm}$. $0,2 \%$ iger $\mathrm{HCl}$ in 20 Stunden eine Fibrinflocke in Lösung. Obgleich die Lösung, aus welcher dasselbe mittelst Dialyse gefällt wurde, keine wahrnehmbare Farbe zeigte, stellte dennoch auch dieses Pepsin ein gelbliches Pulver dar.

Ich erwähnte schon, dass das Pepsin sich, wenn der Säuregehalt der Lösung durch Dialyse allmählich verringert wird, in der Form durchsichtiger Kügelchen absetzt. Das ist auch der Fall, wenn die Fällung durch Ammonsulfat verursacht wird, unter der Bedingung, dass der Niederschlag sich langsam bildet. Hierzu wurde in die Pepsinlösung ein Dialysator mit gesättigter Ammonsulfatlösung und Krystallen in 
Ueberschuss gestellt. Dann und wann wurde die Lösung umgerührt und, sobald sie eine auch beim Umrühren bleibende, nicht allzu geringfügige Trübung zeigte, nach Herausnahme des Dialysators in den Eisschrank gebracht.

Die Kügelchen sind den Globuliten, welche aus Eieralbumin zu erhalten sind, ähnlich, sie sind aber kleiner. Der Diameter ist sehr verschieden, höchstens etwa 15 bis $20 \mu$. Sie sind nicht doppelbrechend. Krystalle habe ich daraus nicht erhalten können.

Unter Ammonsulfatlösung aufbewahrt, kann das Pepsin lange Zeit unverändert bleiben. Ein ganz aus Kügelchen bestehendes, wiederholt mit halbgesättigter Ammonsulfatlösung durch Decantiren gewaschenes Präparat ist jetzt mehr als 4 Jahre in einem starken Temperaturschwankungen ausgesetzten Zimmer unverändert geblieben. Nicht nur lässt sich keine Veränderung bei der mikroskopischen Untersuchung nachweisen, sondern es wirkt auch, vom Ammonsulfat befreit und in Salzsäure gelöst, kräftig eiweissverdauend.

Ich war wohl gezwungen, meine Versuche, aus der Magenschleimhaut einen Stoff von immer derselben Zusammensetzung zu bereiten, aufzugeben, nicht nur, weil bei noch öfterer Wiederholung der Reinigung, welche unvermeidlich mit ziemlich grossem Verlust an Material einhergeht, die Bereitung von für die Analysen ausreichenden Substanzmengen praktisch kaum ausführbar sein würde, sondern in erster Linie, weil ich genügend erfahren hatte, mit wie grosser Zähigkeit das Pepsin einmal aufgenommene Verunreinigungen festhält, und wie leicht dieser höchst complicirte und labile Stoff in salzarmer Lösung, also auch bei der Dialyse, wenn auch in geringem Maasse, zersetzt wird. Deshalb entschloss ich mich, zu versuchen, ob vielleicht der Magensaft des Hundes, welcher jetzt, nach den glänzenden $\mathrm{Pawlow}$ 'schen-Arbeiten, in unbeschränkter Menge und ohne Beimischung zu erhalten ist, befriedigendere Resultate geben würde.

Meine an Herrn Prof. Pawlow gerichtete Bitte, mir über die Operationsmethode nähere Auskunft zu geben, wurde in zuvorkommendster Weise beantwortet. Von Herrn Dr. Walther 
erhielt ich eine sehr ausführliche und genaue Mittheilung in Bezug auf alles, was beim Anlegen einer Magen- und Oesophagusfistel nach der Pawlow'schen Methode in Betracht genommen werden muss. Mein hiesiger College, Herr Prof. Narath, hatte die grosse Freundlichkeit für mich, bei einem $25 \mathrm{~kg}$ schweren Hund erst eine Magenfistel und, als die silberne Canüle fest darin verwachsen war, eine Oesophagusfistel anzulegen.

Es ist mir eine angenehme Pflicht, hier den Herren Pawlow, Walther und Narath für ihre von mir hochgeschätzte Hülfe meinen verbindlichsten Dank zu bringen.

Von Anfang Januar 1901 ab ist der fortwährend kräftige und gesunde Hund regelmässig, gewöhnlich drei Mal in jeder Woche, für die Lieferung von Magensaft gebraucht worden. Bei der Scheinfütterung mit Fleisch wurde im Mittel in der ersten Stunde $200 \mathrm{ccm}$., in der zweiten Stunde noch $100 \mathrm{ccm}$. Magensaft aufgefangen. Nach jeder Viertelstunde wurde die gesammelte Flüssigkeit auf ein Filter gebracht zur Entfernung von Schleimflöckchen. Das Filtrat war beinahe immer vollkommen farblos und klar. Bisweilen, wenn der Pepsingehalt gross und die Temperatur des Zimmers niedrig war, zeigte es eine geringe Opalescenz, welche aber bei Erwärmung auf Körpertemperatur sogleich spurlos verschwand. Bei Abkühlung kehrte dann die Opalescenz wieder. Die Acidität war im Mittel 0,16 norm. HCl oder ein wenig mehr.

Wie vor kurzer Zeit schon von Nencki und Sieber mitgetheilt worden ist, ${ }^{1}$ ) wird aus dem reinen Magensaft des Hundes, genau so wie aus dem mit Salzsäure hergestellten Extract der Magenschleimhaut, ein grosser Theil des Pepsins, sobald der Säuregehalt, am besten mittelst Dialyse, bis auf etwa $0,02 \%$ verringert wird, ausgefällt.

Ich bereitete das Pepsin folgenderweise. Der filtrirte Magensaft wurde etwa 20 Stunden lang bei einer nicht weit über $0^{\circ} \mathrm{C}$. gelegenen Temperatur gegen destillirtes Wasser dialysirt. Dann wurde die trübe Flüssigkeit centrifugirt und

1) Diese Zeitschrift, Bd. XXXII, S. 291. 
der Bodensatz mit einem geringen Quantum der Flüssigkeit (der grössere Theil derselben wurde abgegossen) auf ein kleines Filter gebracht, mit wenig destillirtem Wasser gewaschen, abgepresst, vom Filter abgehoben und im Exsiccator getrocknet.

Ebenso wie das Pepsin aus der Magenschleimhaut des Schweines, setzte auch hier der Stoff sich in durchsichtigen Kügelchen im Dialysator ab. Das Pepsin war aber jetzt vollkommen farblos, auch nach dem Trocknen, wenn nur der Magensaft ganz frei von Farbstoff war. Das war beinahe immer der Fall. Einzelne Male aber ereignete es sich, dass während der Absonderung des Magensaftes ein wenig Darminhalt und damit Gallenfarbstoff in den Magen zurückfloss. So lange der Magensaft deutlich gelb gefärbt war, wurde er nicht für die Pepsinbereitung verwendet. Es stellte sich aber heraus, dass auch die geringsten Spuren vom Gallenfarbstoff vom Pepsin festgehalten werden. Wenn der Magensaft nur noch so wenig davon enthielt, dass die gelbe Farbe nicht mehr mit Sicherheit zu erkennen war, zeigte dennoch das mittelst Dialyse daraus gefällte Pepsin eine gelbliche Farbe, welche beim Trocknen grünlich wurde. Zur Analyse habe ich ausschliesslich völlig farbloses Pepsin verwendet.

Die vom mittelst Dialyse gefällten Pepsin getrennte Flüssigkeit wurde halb gesättigt mit Ammonsulfat. Dadurch wurde wieder ein nicht unbeträchtlicher Niederschlag hervorgerufen, welcher, in oben beschriebener Weise mittelst Dialyse von Salz befreit, in $0,2 \%$ iger $\mathrm{HCl}$ gelöst, durch Dialyse wieder gefällt, abfiltrirt und getrocknet wurde. Die Substanz wurde fein zerrieben aufbewahrt, bis eine zur Analyse genügende Menge zusammengebracht war. Das von Ammonsulfat gefällte Pepsin wurde niemals mit dem mittelst Dialyse aus dem frischen Magensaft bereiteten vermischt.

Zuerst habe ich den Phosphorgehalt untersucht.

Die nähere Untersuchung des aus Schweinemagen erhaltenen Pepsins hatte es mir schon zweifelhaft gemacht, ob der darin gefundene Phosphor thatsächlich von der höchst complicirten Eiweisssubstanz, aus welcher dieses Pepsin hauptsächlich besteht, herstammte. Wie ich früher mittheilte, be- 
trug der Phosphorgehalt meiner ersten Präparate etwa 1\%; ich bemerkte damals aber schon, dass ich eine bald grössere, bald geringere Verunreinigung mit phosphorhaltigen Stoffen für wahrscheinlich hielt. Je mehr nun das Pepsin gereinigt wurde, desto geringer wurde gewöhnlich der P-Gehalt gefunden, obwohl ich das Schweinepepsin nie frei davon fand. Ausserdem war es mir klar geworden, dass. ich mit Unrecht das beim Erhitzen der sauren Pepsinlösung sich ausscheidende Coagulationsprodukt mit den bekannten Nucleoproteiden in eine Gruppe gestellt hatte. Darauf komme ich noch zurück.

Für die Phosphorbestimmung wurde die bei $110^{\circ} \mathrm{C}$. getrocknete Substanz einige Stunden im Kjeldahl-Kölbchen mit einigen Cubikcentimetern starker Salpetersäure, welche dann und wann nachgefüllt wurde, über sehr kleiner Flamme erhitzt. Wenn das Entweichen rother Dämpfe, auch beim Zufliessen neuer Salpetersäure ganz oder so gut wie ganz aufhörte, wurde mit Soda alkalisch gemacht, die Lösung in der Platinschale zur Trockene ausgedampft und die bei $150^{\circ} \mathrm{C}$. getrocknete Substanz geglüht. Die Schmelze wurde in der bekannten $\mathrm{K}^{\top}$ eise mit Molybdänsäure und Ammoniumnitrat und dann mit Magnesiamixtur behandelt.

Jetzt fand ich das Pepsin phosphorfrei. Aus 0,6768 $\mathrm{g}$ mittelst Dialyse gefälltem Pepsin erhielt ich $0,0003 \mathrm{~g} \mathrm{Mg}_{2} \mathrm{P}_{2} \mathrm{O}_{7}$ und aus $0,8622 \mathrm{~g}$ eines anderen in derselben Weise bereiteten Präparates $0,0004 \mathrm{~g} \mathrm{Mg}_{2} \mathrm{P}_{2} \mathrm{O}_{7}$, welches also einem Phosphorgehalt von etwa $0,01 \%$ entsprechen würde.

Es braucht kaum gesagt zu werden, dass diese Spuren von Phosphor einer Verunreinigung zuzuschreiben sind. Ausserdem war es möglich, das Pepsin so zu bereiten, dass auch nicht die geringste Spur von Phosphor darin nachzuweisen war, und zwar aus dem dialysirten und so theilweise von Pepsin befreiten Magensaft. Wie oben erwähnt, wurde daraus mittelst Halbsättigung mit Ammonsulfat aufs Neue Pepsin gefällt, welches dann vom Salz befreit und in $0,2 \%$ iger $\mathrm{HCl}$ aufgelöst und mittelst Dialyse wieder gefällt wurde. Offenbar war von der ersten Pepsinfällung der verunreinigende phosphorhaltige Stoff völlig mitgeschleppt. Aus mehr wie $0,6 \mathrm{~g}$ des 
mittelst Ammonsulfat gefällten Pepsins konnte bei der üblichen Behandlungsweise mit Magnesia-Mixtur kein sichtbarer und wägbarer Niederschlag erhalten werden. Nichtsdestoweniger besass dieses Pepsin eine nicht geringere eiweissverdauende Kraft als das durch Dialyse des Magensaftes bereitete.

Nencki und Sieber fanden das von ihnen aus dem Magensaft des Hundes dargestellte Pepsin phosphorhaltig, selbst wenn das Pepsin mit Alkohol gewaschen und dadurch verändert war. Sie fanden aber den P-Gehalt nicht nur klein, sondern auch in verschiedenen Präparaten sehr verschieden. Im mittelst Dialyse gefällten und nicht ausgewaschenen Pepsin schwankte derselbe zwischen $0,073 \%$ und $0,148 \%$, in den mit Alkohol ausgewaschenen Präparaten zwischen 0,045\% und $0,091 \%$. Diese Forscher nehmen an, dass das Pepsin Lecithin enthält und zwar, theilweise wenigstens, nicht als Verunreinigung, sondern in einer den hauptsächlich von Bing studirten Verbindungen von Lecithin mit Glucose, Morphin u. s.w. ähnlichen Verbindung.

Ich werde die Möglichkeit, dass im Magensaft eine Verbindung von Pepsin mit Lecithin vorkommt, nicht in Abrede stellen; ich betone aber, dass die Existenz des Enzyms nicht von der Anwesenheit von Lecithin oder irgend einer anderen P-haltigen Gruppe im Pepsinmolekül abhängig ist, nachdem ich ein höchst wirksames Pepsin bereitet habe, in dem entweder gar kein, oder nur spurenweise Phosphor aufzufinden war. Verunreinigung des Pepsins mit Phosphor kann, ausser von Lecithin, auch von anderen Stoffen verursacht werden. Der bei der Scheinfütterung gelieferte Magensaft enthält, wenigstens bei dem von mir gebrauchten Hund, immer ein wenig Schleim, welcher durch Filtration leicht zu entfernen ist. Dieser Schleim besteht aus einem P-haltigen Eiweissstoff. Vom Filter genommen und mit Wasser und Alkohol gewaschen, löst derselbe sich so gut wie nicht in verdünnter Salzsäure, mit Pepsin und Salzsäure digerirt, verliert dieses Mucin aber allmählich die gallertige Consistenz und es wird, unter Ausscheidung eines in Alkali leicht löslichen Bodensatzes, aufgelöst. Vermuthlich gehört es also zu den Nucleoproteiden und sicher 
ist die Möglichkeit vorhanden, dass es, während einiger Zeit mit dem Magensaft in Berührung bleibend, theilweise verdaut wird und der Lösung. P-haltige Spaltungsprodukte abgibt. Deshalb habe ich immer jede Viertelstunde den gesammelten Magensaft sogleich auf das Filter gebracht.

In Bezug auf den Chlorgehalt stimmen meine Befunde mit denjenigen von Nencki und Sieber überein. Während diese Forscher, in fünf Bestimmungen, entweder $0,47 \%$ oder $0,48 \%$ Cl fanden, fand ich $0,49 \%$. Den von Nencki und Sieber für die Auffassung, dass das Chlor ein Bestandtheil des Pepsinmoleküls darstellt, angeführten Gründen kann ich einen hinzufügen. Das entweder unmittelbar mittelst Dialyse oder erst mittelst Ammonsulfat gefällte Pepsin wurde, in möglichst grosser Concentration, bei $37^{\circ} \mathrm{C}$. in $1 \%$ iger Oxalsäure gelöst und aus dieser Lösung wieder durch Dialyse gefällt. Der Niederschlag verdaute, in Oxalsäure gelöst, vorzüglich Fibrin. Wenn nun das im aus der Salzsäurelösung ausgeschiedenen Pepsin gefundene Chlor von ungenügender Entfernung der Salzsäure herrührte, so müsste erwartet werden, dass in dem aus der Oxalsäure ausgeschiedenen Pepsin das Chlor nicht mehr zu finden sein würde. Dennoch war jedesmal Chlor ganz deutlich nachzuweisen.

Für die Bestimmung des Schwefelgehalts wurde die Sub$\operatorname{stan} z$ in der von Hammarsten ${ }^{1}$ ) angegebenen Weise, mit der kleinen, bei der Phosphorbestimmung schon erwähnten Modification (Erhitzen über einer kleinen Flamme statt im Wasserbad) mit Salpetersäure erhitzt und nach Zusatz von Soda, getrocknet und über der Alkoholflamme verbrannt. Die Schmelze wurde in Salzsäure gelöst, durch wiederholtes Eindampfen mit starker Salzsäure von Salpetersäure befreit, in $1 \%$ iger $\mathrm{HCl}$ aufgelöst und mit Chlorbaryum versetzt.

Die Kohlenstoff-, Wasserstoff- und Stickstoffbestimmungen hatte mein Assistent, Herr Middelveld-Viersen, die Güte für mich auszuführen. Die C- und H-Bestimmung geschah in der üblichen Weise mit Bleichromat und vorgelegter Kupfer-

1) Diese Zeitschr. Bd. VII, S. 257 und Bd. IX, S. 289. 
spirale, die N-Bestimmung wurde nach der Kjeldahl'schen Methode gemacht, in der von Böthlinck ${ }^{1}$ ) angegebenen Weise, nur mit der kleinen Abweichung, dass beim Titriren Methylorange statt Congoroth als Indicator verwendet wurde.

Die Resultate waren folgende:

I. Pepsin mittelst Dialyse aus dem Magensaft gefällt. $0,1962 \mathrm{~g}$ gibt $0,0002 \mathrm{~g}$ Asche.

a) $0,3077 \mathrm{~g}$ gibt $0,5869 \mathrm{~g} \mathrm{CO}_{2}$ und $0,1989 \mathrm{~g} \mathrm{H}_{2} \mathrm{O}$, also auf aschefreie Substanz: 52,06\% C und 7,19\% $\mathrm{H}$.

b) $0,3116 \mathrm{~g}$ gibt $0,5915 \mathrm{~g} \mathrm{CO}_{2}$ und $0,1987 \mathrm{~g} \mathrm{H}_{2} \mathrm{O}$, also auf aschefreie Substanz: $51,81 \% \mathrm{C}$ und $\mathbf{7 , 0 9} \% \mathrm{H}$.

c) $0,4471 \mathrm{~g}$ gibt $0,8542 \mathrm{~g} \mathrm{CO}_{2}$ und $0,2838 \mathrm{~g} \mathrm{H}_{2} 0$. also auf aschefreie Substanz: $52,13 \% \mathrm{C}$ und $\mathbf{7 , 0 6} \% \mathrm{H}$.

a) $0,3607 \mathrm{~g}$ gibt $14,55 \mathrm{ccm} . \mathrm{n} / 4 \mathrm{NH}_{3}=50,925 \mathrm{mg} \mathrm{N}$, also auf aschefreie Substanz: 14,13\% N.

b) $0,3545 \mathrm{~g}$ gibt $14,5 \mathrm{ccm} . \mathrm{n} / 4 \mathrm{NH}_{3}=50,75 \mathrm{mg} \mathrm{N}$, also auf aschefreie Substanz: $14,33 \% \mathrm{~N}$. $0,4174 \mathrm{~g}$ gibt $0,0507 \mathrm{~g} \mathrm{BaSO}_{4}=0,00696 \mathrm{~g} \mathrm{~S}$, also auf aschefreie Substanz: 1,66\% S.

II. Pepsin bereitet wie I. $0,1676 \mathrm{~g}$ gibt $0,0001 \mathrm{~g}$ Asche. $0,3422 \mathrm{~g}$ gibt $0,6511 \mathrm{~g} \mathrm{CO}_{2}$ und $0,2199 \mathrm{~g} \mathrm{H}_{2} \mathrm{O}$, also auf aschefreie Substanz: $51,92 \% \mathrm{C}$ und $\mathbf{7 , 1 4} \% \mathrm{H}$. $0,3019 \mathrm{~g}$ gibt $12,5 \mathrm{ccm} . \mathrm{n}^{\prime} 4 \mathrm{NH}_{3}=43,75 \mathrm{mg} \mathrm{N}$, also auf aschefreie Substanz: 14,50\%

III. Pepsin bereitet wie I.

$0,2198 \mathrm{~g}$ gibt $0,0002 \mathrm{~g}$ Asche.

$0,3389 \mathrm{~g}$ gibt $0,6472 \mathrm{~g} \mathrm{CO}_{2}$ und $0,2137 \mathrm{~g} \mathrm{H}_{2} \mathrm{O}$, also auf aschefreie Substanz: 52,13\% C und 7,01\% $\mathrm{H}$. $0,3496 \mathrm{~g}$ gibt $14,55 \mathrm{ccm} . \mathrm{n} / 4 \mathrm{NH}_{\mathrm{s}}=50,925 \mathrm{mg} \mathrm{N}$, also auf aschefreie Substanz: 14,58\% N.

$0,7298 \mathrm{~g}$ gibt $0,0869 \mathrm{~g} \mathrm{BaSO}_{4}=0,0119 \mathrm{~g} \mathrm{~S}$, also auf aschefreie Substanz: 1,63\% S.

IV. Pepsin bereitet wie I.

$0,1884 \mathrm{~g}$ gibt $0,0001 \mathrm{~g}$ Asche.

$0,3549 \mathrm{~g}$ gibt $0,6713 \mathrm{~g} \mathrm{CO}_{2}$ und $0,2213 \mathrm{~g} \mathrm{H}_{2} \mathrm{O}$, also auf aschefreie Substanz: 51,61\% $\mathrm{G}$ und $6,93 \% \mathrm{~N}$. $0,3509 \mathrm{~g}$ gibt $14,6 \mathrm{ccm} . \mathrm{n} / 4 \mathrm{NH}_{3}=51,1 \mathrm{mg} \mathrm{N}$, also auf aschefreie Substanz: 14,57\% N. $0,8574 \mathrm{~g}$ gibt $0,1003 \mathrm{~g} \mathrm{BaSO}_{4}=0,0138 \mathrm{~g} \mathrm{~S}$, also auf aschefreie Substanz: 1,61\% S.

1) Arch. des Sciences biol., T. V, p. 176. 
V. Pepsin bereitet durch Halbsättigen des Filtrats des dialysirten Magensaftes mit Ammonsulfat, Dialysiren des Niederschlages gegen $0,2 \%$ ige $\mathrm{HCl}$, Lösen in $0,2 \%$ iger $\mathrm{HCl}$ bei $37^{\circ} \mathrm{C}$., Filtriren, Dialyse gegen Wasser.

$0,2236 \mathrm{~g}$ gibt $0,0004 \mathrm{~g}$ Asche.

$0,3848 \mathrm{~g}$ gibt $0,7369 \mathrm{~g} \mathrm{CO}_{2}$ und $0,2478 \mathrm{~g} \mathrm{H}_{2} \mathrm{O}$,

also auf aschefreie Substanz 52,32\% C und $\mathbf{7 , 1 6} \% \mathrm{H}$.

$0,3480 \mathrm{~g}$ gibt $14,65 \mathrm{ccm} . \mathrm{n} / 4 \mathrm{NH}_{3}=51,275 \mathrm{mg} \mathrm{N}$,

also auf aschefreie Substanz: 14,75\% $\%$.

$0,3656 \mathrm{~g}$ gibt $0,0489 \mathrm{~g} \mathrm{BaSO}_{4}=0,0067 \mathrm{~g} \mathrm{~S}$,

also auf aschefreie Substanz: $1,83 \% \mathrm{~S}$.

VI. Pepsin bereitet wie V.

Aschebestimmung verunglückt.

$0,3065 \mathrm{~g}$ gibt $0,5846 \mathrm{~g} \mathrm{CO}_{2}$ und $0,1938 \mathrm{~g} \mathrm{H}_{\mathbf{2}} \mathrm{O}$,

also, ohne Berücksichtigung der Asche: $\mathbf{5 2 , 0 1} \% \mathrm{C}$ und $\mathbf{7 , 0 2} \% \mathbf{H}$.

$0,3523 \mathrm{~g}$ gibt $14,75 \mathrm{ccm} . \mathrm{n} / 4 \mathrm{NH}_{3}=51,625 \mathrm{mg} \mathrm{N}$,

also, ohne Berücksichtigung der Asche: 14,65\% N.

Zur Erleichterung der Uebersicht stelle ich die erhaltenen Zahlen in folgender Tabelle zusammen:

\begin{tabular}{|c|c|c|c|c|c|}
\hline & G & $\mathrm{H}$ & $\mathrm{N}$ & $\mathrm{S}$ & \\
\hline & 52,13 & 7,06 & 1413 & \multirow{4}{*}{1,66} & \multirow{6}{*}{$\begin{array}{l}\text { Pepsin mittelst Dialyse des Magen- } \\
\text { saftes gefällt. }\end{array}$} \\
\hline I. & 52,06 & 7,19 & 14,33 & & \\
\hline & 51,81 & 7,09 & & & \\
\hline II. & 51,92 & 7,14 & 14,50 & & \\
\hline III. & 52,13 & 7,01 & 14,58 & 1,63 & \\
\hline IV. & 51,61 & 6,93 & 14,57 & 1,61 & \\
\hline V. & 52,32 & 7,16 & 14,75 & 1,83 & Pepsin aus dem dialysirten Magensaft \\
\hline VI. & 52,01 & 7,02 & 14,65 & & mittelst Ammonsulfat gefällt. \\
\hline
\end{tabular}

Dass der Schwefelgehalt in $V$ ein wenig höher als in den anderen Präparaten gefunden wurde, ist vielleicht durch Verunreinigung mit Ammonsulfat zu erklären. Diese Annahme ist mit dem $\mathrm{N}$-Gehalt in Uebereinstimmung. Denn $0,2 \%$ von $\left(\mathrm{NH}_{4}\right)_{2} \mathrm{SO}_{4}$ herrührendem $\mathrm{S}$ entspricht $0,175 \% \mathrm{~N}$. Der $\mathrm{N}-$ Gehalt würde also von 14,75 auf 14,575 kommen und so besser mit dem N-Gehalt der anderen Präparate übereinstimmen. 
Die Menge des Präparates VI reichte zu einer Schwefelbestimmung nicht aus. Dass hier der Aschegehalt nicht berücksichtigt werden konnte, verursacht wohl keinen in Betracht kommenden Fehler, nachdem ja der Aschegehalt immer sehr gering war.

Frau Schoumow-Simanowski ${ }^{1}$ fand für das mittelst Abkühlung aus dem Magensaft des Hundes gefällte und mit Alkohol gewaschene Pepsin:

$$
\text { C. } 50,71, \mathrm{H} 7,17
$$

und für das durch Sättigung des Magensaftes mit Ammonsulfat gefällte:

$$
\text { C } 50,37, \mathrm{H} 6,88, \mathrm{~N}\left\{\begin{array} { l } 
{ 1 4 , 5 5 } \\
{ 1 5 , 0 , }
\end{array} \mathrm { S } \left\{\begin{array}{l}
1,35 \\
1,24,
\end{array}\right.\right.
$$

wobei der Aschegehalt nicht in Rechnung gebracht wurde.

Nencki und Sieber fanden bei der Analyse der mittelst Dialyse des Magensaftes gefällten Substanz:

$$
\text { C. 51,26, H 6,74, N 14,33, S 1,5, }
$$

während ich C. 51,99, H 7,07, N 14,44, S 1,63

als den mittleren Werth fand bei der Analyse der vier mittelst Dialyse des Magensaftes erhaltenen Präparate.

Der Unterschied betrifft in erster Linie den C-Gehalt, welcher von mir in allen Fällen höher gefunden wird als von den russischen Forschern, obgleich meine Zahlen den (in einer Analyse) von Nencki und Sieber gefundenen sehr nahe kommen. Mit Rücksicht auf das Fehlen von Phosphor in meinen Präparaten und auch mit Rücksicht auf den Aschegehalt (N. und S. fanden $0,57 \%$ Asche) glaube ich die von mir bereitete Substanz für am besten gereinigt halten zu dürfen. In dem aus der Magenschleimhaut des Schweines bereiteten Pepsin, welches ich nicht so reinigen konnte, dass es phosphorfrei wurde, fand ich auch den C-Gehalt niedriger, schwankend zwischen 48,18 und 50,77.

Den Aschegehalt fand ich immer sehr niedrig, zu etwa $0,1 \%$ im durch Dialyse des Magensaftes gefällten, $0,2 \%$ im mit Hülfe von Ammonsulfat bereiteten Pepsin. In Ueberein-

1) Arch. d. Sciences biol., T. II, p. 463. 
stimmung mit Nencki und Sieber konnte ich Eisen in der Asche nachweisen. Eisenbestimmungen habe ich nicht gemacht.

Dass das beim Erhitzen der sauren Lösung dieses Pepsins sich ausscheidende Spaltungsprodukt kein Phosphor enthält, ist selbstverständlich. Auch den beim Erhitzen des frischen Magensaftes ausfallenden Körper fand ich aber, wenn derselbe gehörig erst mit Wasser und dann mit Alkohol und Aether gewaschen war, phosphorfrei. Es ist also nicht erlaubt, diesen Eiweisskörper in die Gruppe der Nucleoproteide einzureihen.

In Bezug auf die Ausscheidung dieses Stoffes habe ich früher mitgetheilt, dass dieselbe nur dann richtig stattfindet, wenn die saure Lösung des Pepsins schnell, über der Flamme, erhitzt wird. Wird die Lösung, im Wasserbad oder im Luftbad, langsam erhitzt, so kann sie, selbst bei der Kochhitze, vollkommen klar bleiben, um so leichter, je weniger Säure sie enthält. Dass für die Bildung des Niederschlages Erhitzen, und zwar schnelles Erhitzen der Lösung nicht genügend, sondern dass auch saure Reaction erforderlich ist, habe ich schon im Anfang meiner Untersuchungen über Pepsin gefunden, und etwas Anderes ist, wie ich glaube, aus dem von mir in Bezug hierauf Mitgetheilten nicht abzuleiten. Deshalb verwunderte mich die Behauptung Friedenthal's: «Entgegen den Angaben von Pekelharing enthält der Hundemagensaft keinen durch Kochen fällbaren Eiweisskörper. Wohl erhält man beim Kochen des frischen Magensaftes eine flockige Fällung, aber diese Fällung wird durch die Salzsäure des Magensaftes in der Hitze bewirkt, nicht durch die Siedehitze allein.») Thatsächlich hat Friedenthal durch seine Beobachtung nur meine Mittheilung bestätigt, ohne aber den Erfolg der langsamen Erhitzung zu beachten.

Hinsichtlich einer anderen Frage muss ich Friedenthal vollkommen Recht geben. Die Richtigkeit seiner, inzwischen auch von Nencki und Sieber bestätigten, Beobachtung, dass aus dem beim Erhitzen sich ausscheidenden Eiweissstoff ein

1) Engelmann's Archiv f. Physiol., 1900, S. 186. 
Kohlehydrat, und zwar eine Pentose zu erhalten ist, kann nicht bezweifelt werden. Dass ich früher, nach dem Kochen mit Schwefelsäure, eine reducirende Substanz nicht fand, muss ich als einen Fehler bezeichnen. Einmal habe ich aus der reducirenden und die Phloroglucinreaction gebenden, von Eiweiss befreiten Lösung, nach Behandlung mit Phenylhydrazin und Natriumacetat, bei Abkühlung sich ausscheidende aus feinen Krystallnadeln zusammengesetzte Rosetten erhalten, jedoch in für Schmelzpunktbestimmung $\mathrm{zu}$ geringen Mengen. Gewöhnlich setzte das Osazon sich in kleinen, gelben, durchsichtigen Kügelchen ab. Für eine nähere Untersuchung dieses Kohlehydrates wäre es nöthig, erst grössere Mengen des Gerinnungsproduktes zu bereiten. Dasselbe gilt in Bezug auf die Untersuchung der beim Kochen mit Säure aus diesem Stoff frei werdenden Purinbasen. Dem früher darüber Mitgetheilten - welches sowohl von Friedenthal wie von Nencki und Sieber bestätigt worden ist - habe ich jetzt nichts mehr hinzuzufügen, als dass ich aus dem Pepsin des Schweines eine Basis bereitet habe, welche als Xanthin charakterisirt werden konnte. Die hierauf bezüglichen Fragen müssen, ebenso wie die in Bezug auf die anderen, bei der Erhitzung aus dem Pepsin entstehenden Spaltungsprodukte, der weiteren Forschung überlassen bleiben, welche übrigens, u. A. weil die Bereitung genügender Mengen des Ausgangsmaterials sehr viel Zeit und Arbeit in Anspruch nimmt, auf nicht geringe Schwierigkeiten stösst.

Einen der aus dem Gerinnungsprodukt - wie es doch wohl erlaubt sein wird, den beim Erhitzen der sauren Pepsinlösung (der oxalsauren, ebenso gut wie der salzsauren) sich ausscheidenden Niederschlag zu nennen - durch Spaltung zu erhaltenden Stoffe habe ich etwas besser kennen gelernt.

Das Gerinnungsprodukt wurde durch schnelle Erhitzung von circa $75 \mathrm{ccm}$. des Magensaftes zugleich über einer.grossen Flamme zur Ausscheidung gebracht. Beim Abkühlen setzt sich der Niederschlag gut ab. Derselbe wurde erst durch Decantiren mit Wasser gewaschen, auf das Filter gebracht, sorgfältig mit Alkohol und Aether ausgewaschen und getrocknet. 
Dieser Stoff wird auch aus dem künstlichen Magensaft des Schweines völlig farblos erhalten.

Dass derselbe nicht als ein Nucleoproteid betrachtet werden darf, wenn auch ein in Säure unlöslicher, in Alkali leicht löslicher Eiweissstoff, aus welchem durch Kochen mit mineralen Säuren Purinbasen und Pentose abzuspalten sind, das war mir schon, bevor ich wusste, dass dieser Stoff phosphorfrei zu bereiten ist, wahrscheinlich geworden, nachdem alle Versuche, eine Nucleinsäure daraus darzustellen, fehlgeschlagen. Bei der Behandlung mit Alkali lieferte das Gerinnungsprodukt zwar eine Säure, diese Säure aber war ein Eiweissstoff, wenig löslich in Wasser, unlöslich in verdünnter Säure und leicht löslich in heissem Alkohol.

Die Bereitung dieser Substanz fand am besten in folgender Weise statt. Das Gerinnungsprodukt wird in 1\% iger Kalilauge gelöst und dann 5 Minuten im Wasserbad gekocht. Die Anfangs farblose Lösung wird beim Kochen gelblich gefärbt. - Sie wird dann mit Salzsäure angesäuert. In Folge dessen bildet sich, unter Schwefelwasserstoffentwickelung, ein reichlicher, rein weisser Niederschlag, welcher abfiltrirt wird. Das Filtrat ist ebenso farblos, wird aber wieder gelb, sobald die Reaction alkalisch gemacht wird; es gibt die Biuretreaction und liefert, nach Kochen mit Salzsäure, Pentose.

Der Niederschlag wird mit 0,5\% iger Salzsäure ausgewaschen, in kochendem 85\% igen Alkohol gelöst und heiss filtrirt. Beim Abkühlen setzt, sich der Stoff als eine gallertige Masse ab; allmählich bildet sich an der Oberfläche der Gallerte eine klare Alkoholschicht, welche nur wenig der Substanz in Lösung hält. Die Gallerte wird erst durch Decantiren mit 96\% igem Alkohol gewaschen und dann mit gleichen Theilen Alkohol und Aether angerührt. Der Niederschlag, welcher durch den Aetherzusatz flockig wird und sich gut absetzt, wird dann auf das Filter gebracht, mit reinem Aether gewaschen und getrocknet, wobei bisweilen die Anfangs rein weisse Farbe einen Stich ins Gelbliche bekommt.

Dieser Stoff hat die Eigenschaften einer Säure. In Wasser löst er sich, bei vorsichtigem Zusatz von Alkali, mit saurer 
Reaction. Wird die saure Lösung, zur Entfernung beigemischter Salze, ein paar Tage lang gegen oft erneuertes destillirtes Wasser dialysirt und dann in der von Huiskamp beschriebenen Weise $^{1}$ ) unter einer Spannung von etwa 50 Volt der Elektrolyse unterworfen, so wird das Eiweiss nach der Anode geführt und dort als eine Gallerte ausgeschieden, während das Alkali sich an der Kathode anhäuft.

Ich schlage für diesen Eiweissstoff den Namen Pepsinsäure vor.

Aus der alkoholischen Lösung scheidet diese Säure sich beim Verflüchtigen des Alkohols als eine firnissartige Substanz ab. Sie gibt die Biuret- und die Xanthoproteinreaction und die Reactionen von Adamkiewicz und von Millon. Begreiflicher Weise gibt sie beim Kochen mit Alkali keinen Schwefel ab. Durch anhaltendes Kochen mit Alkali wird sie weiter verändert. Salzsäurezusatz ruft dann keinen Niederschlag mehr hervor.

Aus durch allmähliche Erhitzung veränderten, beim Erhitzen klar gebliebenen Pepsinlösungen ist dieser Stoff nicht mehr zu erhalten.

Die Pepsinsäure habe ich als ein Spaltungsprodukt des aus Schweinemagen bereiteten Pepsins kennen gelernt und später ganz in derselben Weise aus dem Pepsin des Hundes erhalten. Nur habe ich die Pepsinsäure des Schweines, obgleich dieselbe aus dem farblosen Gerinnungsprodukt bereitet wurde, nie ganz farblos darstellen können. Deshalb habe ich für die Elementaranalyse ausschliesslich rein weisse Pepsinsäure des Hundes gebraucht.

Die Resultate der Analyse waren folgende:

I. $0,2676 \mathrm{~g}$ gibt $0,0049 \mathrm{~g}$ Asche, also $1,83 \%$. $0,3548 \mathrm{~g}$ gibt $0,6495 \mathrm{~g} \mathrm{CO}_{8}$ und $0,2156 \mathrm{~g} \mathrm{H}_{2} \mathrm{O}$, also auf aschefreie Substanz 50,81 \% C und 6,87 \% H. 0,3384 g"gibt $13,55 \mathrm{ccm}$. n/4 $\mathrm{NH}_{3}=47,425 \mathrm{mg} \mathrm{N}$, also auf aschefreie Substanz 14,27\% N. $0,7384 \mathrm{~g}$ gibt $0,0568 \mathrm{~g} \mathrm{BaSO}_{4}=0,0077 \mathrm{~S}$, also auf aschefreie Substanz 1,06\% S.

1) Diese Zeitschrift, Bd. XXXIV, S. 32. 
II. $0,3060 \mathrm{~g}$ gibt $0,0068 \mathrm{~g}$ Asche, also $2,2 \%$. $0,3461 \mathrm{~g}$ gibt $0,6332 \mathrm{~g} \mathrm{CO}_{2}$ und $0,2127 \mathrm{~g} \mathrm{H}_{2} \mathrm{O}$.

also auf aschefreie Substanz 51,03\% $\mathrm{C}$ und 6,98 \% $\mathrm{H}$. $0,3393 \mathrm{~g}$ gibt $13.85 \mathrm{ccm} . \mathrm{n} / 4 \mathrm{NH}_{3}=48,475 \mathrm{mg} \mathrm{N}$, also auf aschefreie Substanz 14,61\% N. $0,6504 \mathrm{~g}$ gibt $0,0531 \mathrm{~g} \mathrm{BaSO}_{4}=0,00729 \mathrm{~g} \mathrm{~S}$, also auf aschefreie Substanz 1,14\% S.

III. $0,3024 \mathrm{~g}$ gibt $0,0040 \mathrm{~g}$ Asche, also $1,3 \%$. $0,3509 \mathrm{~g}$ gibt $0,6417 \mathrm{~g} \mathrm{CO}_{2}$ und $0,2251 \mathrm{~g} \mathrm{H}_{2} \mathrm{O}$, also auf aschefreie Substanz 50,53\% C und 7,22\% $\mathrm{H}$.

Wahrscheinlich würde es durch längeres Auswaschen mit 0,5\% iger HCl, zur Entfernung des bei der Ausscheidung des Stoffes gebildeten KCl gelingen, den Aschegehalt herabzudrücken.

Auch in der procentischen Zusammensetzung stimmt also die Pepsinsäure mit den Eiweissstoffen überein. In Bezug auf den C-, H-, N- und S-Gehalt unterscheidet sie sich von dem Gerinnungsprodukt, aus welchem sie hervorgegangen ist, hauptsächlich hinsichtlich des Schwefels.

Für das Gerinnungsprodukt wurde gefunden:

I. $0,3544 \mathrm{~g}$ gibt $0,0005 \mathrm{~g}$ Asche, also $0,14 \%$. $0,3503 \mathrm{~g}$ gibt $0,6458 \mathrm{~g} \mathrm{CO}_{2}$ und $0,2198 \mathrm{~g} \mathrm{H}_{2} \mathrm{O}$, also auf aschefreie Substanz 50,35\% $\mathrm{C}$ und 6,98\% $\mathrm{H}$. $0,3481 \mathrm{~g}$ gibt $14,8 \mathrm{ccm} . \mathrm{n} / 4 \mathrm{NH}_{3}=51,8 \mathrm{mg} \mathrm{N}$, also auf aschefreie Substanz 14,90\% N.

$0,4524 \mathrm{~g}$ gibt $0,0528 \mathrm{~g} \mathrm{BaSO}_{4}=0,00725 \mathrm{~S}$, also auf aschefreie Substanz 1,6\% S.

II. $0,5340 \mathrm{~g}$ gibt $0,0007 \mathrm{~g}$ Asche, also $0,13 \%$. $0,9710 \mathrm{~g}$ gibt $0,1191 \mathrm{~g} \mathrm{BaSO}_{4}=0,01636 \mathrm{~g} \mathrm{~S}$, also auf aschefreie Substanz 1,68\% $\mathrm{S}$.

$\begin{array}{ccccc} & \text { C } & \text { H } & \text { N } & \text { S } \\ \text { Also: Gerinnungsprodukt } & 50,35 & 6,98 & 14,90 & 1,64 \\ \text { Pepsinsäure (im Mittel) } & 50,79 & 7,02 & 14,44 & 1,08\end{array}$

Das aus der Magenschleimhaut des Schweines bereitete Pepsin darf mit dem im Magensaft des Hundes vorhandenen, wie ich glaube, in eine Linie gestellt werden. Wenn auch die Möglichkeit, dass irgend ein Unterschied zwischen beiden 
Stoffen aufzufinden sein wird, offen gelassen werden muss, so dürfen die wenigen bis jetzt gefundenen Unterschiede der Schwierigkeit, die aus der Magenschleimhaut extrahirte Substanz zu reinigen, zugeschrieben werden. Dieselbe konnte niemals ganz farblos erhalten werden und die Analyse der einzelnen Präparate lieferte nicht genügend übereinstimmende Zahlen. Ich beschränke mich auf die Mittheilung der grössten und der kleinsten Werthe, welche bei der Analyse von 5 möglichst sorgfältig gereinigten Präparaten gefunden wurden:

$\begin{array}{lccccc} & \mathrm{C} & \mathrm{H} & \mathrm{N} & \mathrm{P} & \mathrm{S} \\ \text { Maximum } & 50,77 & 7,27 & 15,06 & 0,75 & 1,6 \\ \text { Minimum } & 48,18 & 6,72 & 14,02 & 0,425 & 1,45\end{array}$

Uebrigens war die Uebereinstimmung in Bezug auf die Eigenschaften und auf die Spaltungsprodukte, soweit meine Beobachtung reichte, vollständig.

Beide Stoffe sind, wie alle Eiweissstoffe, linksdrehend. Irgend einen Einfluss der Reaction der Lösung auf die Grösse der Drehung habe ich nicht nachweisen können. Genaue Bestimmungen des Drehungsvermögens habe ich bis jetzt noch nicht ausführen können. Bei Mangel eines Polarisationsapparates, welcher gestattet, die Flüssigkeit anders als bei Zimmertemperatur zu untersuchen, habe ich mich, weil die Löslichkeit des Pepsins bei Zimmertemperatur viel geringer ist als bei $37^{\circ} \mathrm{C}$., für die Bestimmung mit Lösungen geringer Concentration begnügen müssen. Ich glaube aber annehmen zu dürfen, dass $\alpha_{D}$ nicht weit von $50^{\circ}$ entfernt liegt.

Wenn in Betracht genommen wird, dass die aus dem Magensaft des Hundes bereitete Substanz in, für einen Eiweissstoff befriedigender Reinheit erhalten ist, scheint mir die Annahme, dass dieses äusserst wirksame Pepsin das Enzym selbst darstellt und nicht seine Wirkung an Beimischungen verschuldet ist, nicht sehr gewagt mehr.

Erstens wird, wie schon betont wurde, die Wirksamkeit aufgehoben durch Erhitzung, genau bei derselben Temperatur, bei welcher der Eiweissstoff zersetzt wird.

Zweitens verliert der Magensaft, sobald derselbe durch Halbsättigen mit Ammonsulfat dieses Stoffes beraubt wird, 
völlig seine verdauende Wirkung. Bei dem Nachweis dieser Thatsache muss im Auge behalten werden, dass die Anwesenheit von Ammonsulfat für die Pepsinwirkung in hohem Grad hinderlich ist. Ich habe mich aber wiederholt davon überzeugt, dass der vom Ammonsulfatniederschlag abfiltrirte Magensaft, auch wenn das Salz mittelst Dialyse bis auf Spuren entfernt war, nicht die geringste verdauende Kraft besass. Bedenkt man, dass 0,001 mg der von mir bereiteten Substanz in $6 \mathrm{ccm}$. $0,2 \%$ iger $\mathrm{HCl}$ noch zwar schwache, aber doch unverkennbare Einwirkung zeigt auf Fibrin, so ist man wohl zu der Folgerung berechtigt, dass das von Salz befreite Filtrat gar kein Enzym enthält, dass also alles Enzym in den vom Ammonsulfat hervorgerufenen Niederschlag übergegangen ist. Und dieser Niederschlag besteht eben aus dem Eiweissstoff, welcher die Enzymwirkung im höchsten Maasse besitzt.

Einen anderen Grund für meine Auffassung finde ich in der Beobachtung, dass die verdauende Kraft des Magensaftes mit der Menge des durch Erhitzung daraus zu erhaltenden Gerinnungsproduktes gleichen Schritt hält. Mit besonderer Deutlichkeit tritt dies hervor, wenn die Absonderung des Magensaftes beim Hund während der Scheinfütterung mittelst eines Alkoholclysma gefördert wird. Der unter dem Alkoholeinfluss secernirte Magensaft ist grösser in Menge und wenigstens ebenso reich, gewöhnlich aber ein wenig reicher an Salzsäure als der zuvor abgesonderte, dagegen ärmer an Pepsin. Dann scheidet sich auch beim Erhitzen das Gerinnungsprodukt, dessen Menge, wenn die Erhitzung nur schnell stattfindet, als ein Mass des von mir für das Enzym gehaltenen Stoffes zu betrachten ist, in merkbar geringerer Quantität aus. Die relative Menge des Pepsins wurde nach der Mett'schen Methode bestimmt; für die Bestimmung des Gerinnungsproduktes wurden je $50 \mathrm{ccm}$. in einem Kölbchen von $100 \mathrm{ccm}$. Inhalt rasch über der Flamme gekocht und nach Abkühlung auf ein tarirtes Filter gebracht. Der Niederschlag wurde der Reihe nach mit Wasser, Alkohol und Aether gewaschen, bei $110^{\circ} \mathrm{C}$. getrocknet und gewogen. 
So fand ich:

\begin{tabular}{|c|c|c|c|}
\hline \multirow{3}{*}{ I. $\left\{\begin{array}{l}\text { a) } \\
\text { b) }\end{array}\right.$} & \multicolumn{2}{|c|}{ Eiweiss verdaut: } & Gerinnungspro \\
\hline & 3,8 & $\mathrm{~nm}$ & $0,0508 \mathrm{~g}$ \\
\hline & 3 & $»$ & 0,0298 \\
\hline ( a) & 2,6 & 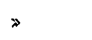 & 0,0384 , \\
\hline (b) & 2,4 & > & 0,0282 \\
\hline (a) & 2,7 & » & 0,0604 \\
\hline 1II. $(\mathrm{b})$ & 2,1 & » & 0,0320 \\
\hline ( a) & $7, \tilde{5}$ & , & 0,0882 \\
\hline (b) & 6 & 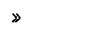 & 0,0837 \\
\hline
\end{tabular}

Nach der Schütz-Borissow'schen Regel ist das Quantum des Pepsins dem Quadrat der Anzahl der Millimeter verdauten Eiweisses proportional. Die für das Gewicht des Gerinnungsproduktes gefundenen Zahlen stimmen zu dieser Regel ziemlich gut, ausser in Versuch IV, wo die Menge des beim Kochen erhaltenen Niederschlages in b nicht gering genug gefunden wurde im Verhältniss zur Verdauungsgeschwindigkeit.

Dabei muss im Auge behalten werden, dass das Mett'sche Verfahren auf grosse Genauigkeit keinen Anspruch macht und dass auch die Bestimmung des Gerinnungsproduktes nicht fehlerfrei ist.

Dass es möglich ist, Pepsinlösungen zu bereiten, welche Eiweiss kräftig verdauen und dennoch die Reactionen von Eiweiss nicht zeigen, ist, wie ich früher schon ausgeführt habe und wie neuerdings auch von Nencki und Sieber betont worden ist, kein Grund, die Eiweissnatur des Pepsins zu verneinen.

Von Bliss und Novy ${ }^{1}$ ) ist eine Beobachtung veröffentlicht, welche Zweifel veranlassen kann, ob man berechtigt ist, das Pepsin als eine Eiweisssubstanz zu betrachten. Diese Forscher fanden nämlich, dass das Pepsin gar nicht angegriffen wird von Formaldehyd, welches doch verschiedene Eiweissstoffe in eine unlösliche Form überzuführen im Stande ist. Die Richtigkeit der Beobachtung kann ich bestätigen. Mit Formol zu einem Gehalt von 2 bis 3\% versetzte Lösungen von Pepsin in Salzsäure können ohne merkbaren Verlust an verdauender Wirkung Tage lang aufbewahrt werden. Selbst-

1) Journal of exp. med., Vol. IV, p. 47. 
verständlich muss beim Prüfen der Wirkung der Gehalt an Formaldehyd mittelst Verdünnung oder mittelst Dialyse beträchtlich herabgesetzt werden, damit nicht das Fibrin selbst angegriffen und für die Verdauung ungeeignet gemacht werde.

Nicht richtig aber ist die Annahme, dass alle Eiweissstoffe vom Formaldehyd angegriffen werden. Insbesondere der hier erwähnte Eiweissstoff aus dem Magensaft ist gegen dieses Reagens unempfindlich. Ich habe die gereinigte Substanz unter Formolzusatz in 0,2\%!oiger Salzsäure gelöst und dann wieder mittelst Dialyse gegen Wasser oder mittelst Ammonsulfat fällen können, ohne dass sich in den Eigenschaften des Pepsins irgend eine Veränderung nachweisen liess.

Der Stoff besitzt nicht nur das Vermögen, in saurer Lösung Eiweiss zu verdauen, sondern er bringt auch, wie ich früher mittheilte, Milch zur Gerinnung. In Uebereinstimmung mit Nencki und Sieber fand ich auch, dass sie in concentrirten Albumoselösungen eine Fällung von "Plastein» hervorruft. Auf Fett übt dieselbe, weder in saurer noch in neutraler oder alkalischer Lösung irgend einen wahrnehmbaren Einfluss aus, obgleich am Magensaft des von mir gebrauchten Hundes die von Vollhard ${ }^{1}$ ) beschriebene fettspaltende Wirkung sich sehr deutlich nachweisen liess.

Den klaren Ausführungen von Nencki und Sieber zu Gunsten der Vorstellung, dass ein und dasselbe Molekül verschiedene Enzymwirkungen haben kann, habe ich nichts hinzuzufügen. Ich schliesse mich vollkommen dieser Auffassung an. Um bei dem bekannten von E. Fischer gebrauchten Bild zu bleiben: es gibt Schlüssel, welche aus einem Ring bestehen, mit verschiedenen angehefteten Seitenstücken, deren jedes zu einem anderen Schloss passt. Sollten auch ein oder mehrere dieser Seitenstücke verbogen oder auf andere Weise unbrauchbar gemacht werden, so können doch die übrig bleibenden noch beim Aufschliessen Dienste leisten.

1) Münch. med. Wochenschr., 1901, S. 141 und Zeitschr. f. klin. Med., Bd. XLII, S. 414. 